\title{
Artificial Phospholipids and Their Vesicles
}

\author{
Andreas Zumbuehl*io \\ Department of Chemistry, University of Fribourg, Chemin du Musée 9, 1700 Fribourg, Switzerland
}
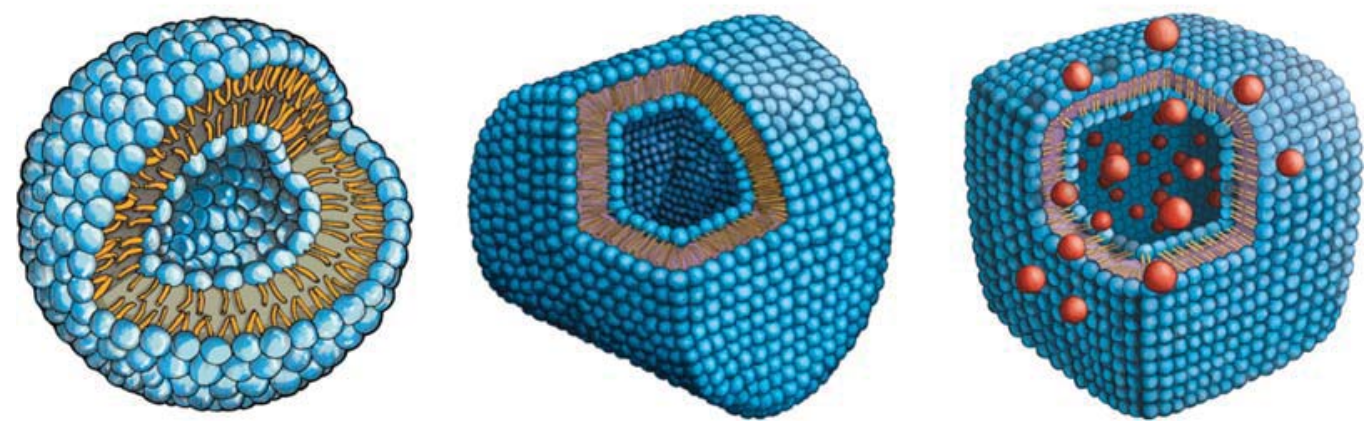

ABSTRACT: Phospholipids are at the heart and origin of life on this planet. The possibilities in terms of phospholipid selfassembly and biological functions seem limitless. Nonetheless, nature exploits only a small fraction of the available chemical space of phospholipids. Using chemical synthesis, artificial phospholipid structures become accessible, and the study of their biophysics may reveal unprecedented properties. In this article, the recent advances by our work group in the field of chemical lipidology are summarized. The family of diamidophospholipids is discussed in detail from monolayer characterization to the formation of faceted vesicles, culminating in the template-free self-assembly of phospholipid cubes and the possible applications of vesicle origami in modern personalized medicine.

\section{INTRODUCTION}

Phospholipids are life-enabling molecules. Over the millennia, nature has created this class of amphiphilic molecules to help bridge interfaces such as the interior and exterior of a cell. ${ }^{1}$ In recent years, it has been more and more appreciated that the phospholipids are not merely limited to a passive amphiphilic role $^{2}$ but are actively involved in human metabolism. ${ }^{3}$ Seemingly small changes in the chemical structure of a phospholipid may decide the life or death of an organism, such as the end functionalization of a long fatty acyl chain from straight to iso-branched. ${ }^{4}$ The study of such structural changes requires the convergence 5 of chemical synthesis and the biochemical and biophysical characterization of phospholipids. ${ }^{6}$ Lipidomics discovers natural phospholipids. The field that forages for nonnatural phospholipids we have termed chemical lipidology. The chemical space to be explored is nearly infinite, and chemical lipidology has the potential to discover unprecedented approaches to nanomedicial applications. In this feature article, I have therefore highlighted several recent examples of chemical lipidology from our work group, expanding on research that began 10 years ago.?

\section{DIFFERENT PARTS OF A PHOSPHOLIPID}

Chemically speaking, a phospholipid is made from four different parts (Figure 1): two fatty acyl chains, a phosphorylated headgroup, and a backbone holding everything together. Each of these four parts can be chemically modified, so the possibilities of putting together new types of

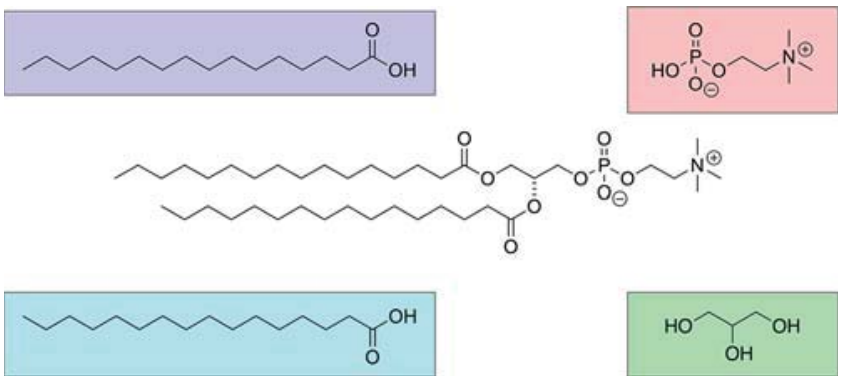

Figure 1. Glycerophospholipids are modular structures made from four building blocks: two fatty acyl chains, a phosphorylated headgroup, and a backbone holding everything together.

phospholipid molecules are nearly endless. ${ }^{7,8}$ In recent decades, the convenience of commercially available phospholipids led to a decrease in the types of phospholipids studied. This was not always the case, and the 1970s and 1980s saw a variety of groups working in the field of chemical lipidology. The medicinal aspects were important from the beginning, and ways to inhibit the enzyme phospholipase $A_{2}$ were sought. ${ }^{9}$ Phospholipase $\mathrm{A}_{2}$ is involved in the homeoviscous adaptation of phospholipids, the cleavage of fatty acids, and the attachment of other fatty acids in order to adapt the cell 

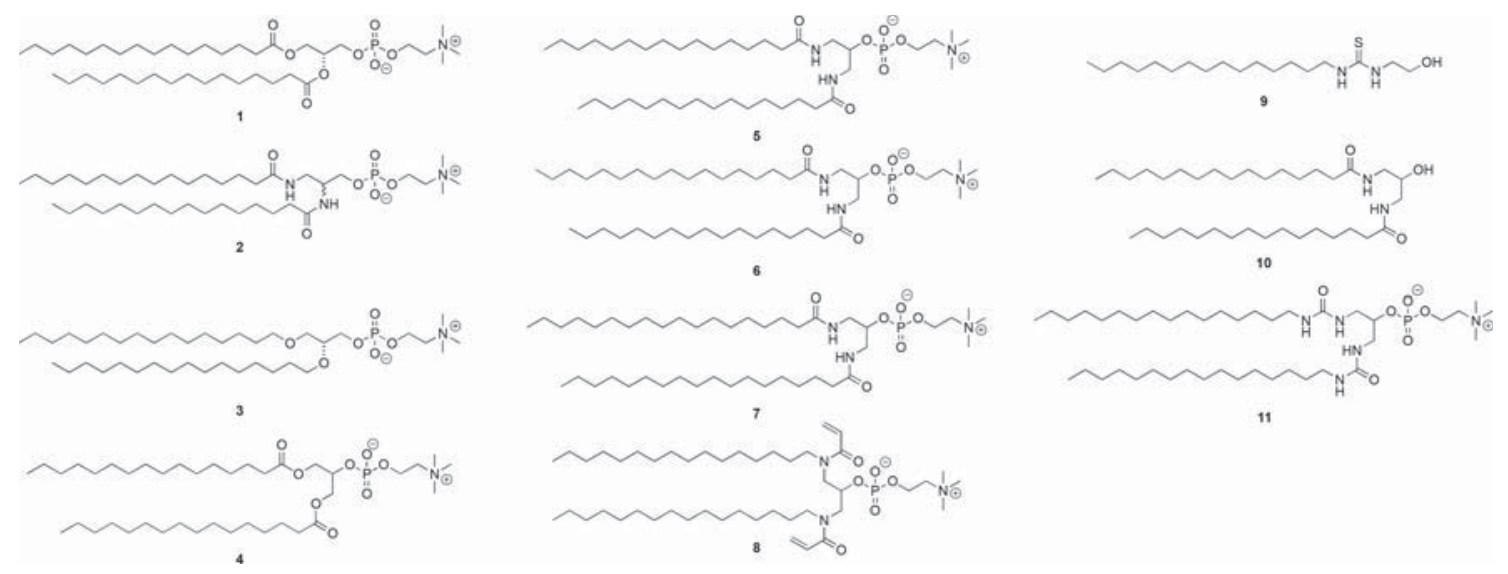

Figure 2. Chemical structures of the lipids discussed in this article.

membrane to external conditions. ${ }^{10}$ Later, the secretory phospholipase $\mathrm{A}_{2}$ of cancer cells was used to cleave an anticancer prodrug chemically attached to the $s n-2$ position of a phospholipid. ${ }^{11}$ Artificial phospholipids were also tested as anti-AIDS drugs, but with limited success. ${ }^{12}$ And one should not forget a series of great minds working on stabilizing liposomes using diacetylene-type phospholipids. ${ }^{13,14}$

\section{PHOSPHOLIPID NOMENCLATURE}

Given the modularity of the phospholipids, there are a nearly infinite number of possible structures that are accessible. Already $>43000$ natural structures are known, and with the advent of lipidomics, ${ }^{15}$ the Lipid MAPS project started its important work of collecting and curating all data and mapping the lipids using a 12-digit identifier code. 3,16

The standard phospholipid nomenclature has been introduced by the International Union of Pure an Applied Chemistry and is based on the Fischer projection of the phospholipid's glycerol backbone. ${ }^{17}$ The fatty acyl chain on the secondary alcohol is brought into an $\mathrm{L}$ position. The numbering of the glycerol carbon centers from top (1) to bottom (3) leads to stereospecific nomenclature (sn) and names such as 1-palmitoyl-2-oleoyl-sn-glycero-3-phosphocholine (POPC) and 3-palmitoyl-2-oleoyl-sn-glcero-1-phosphocholine for its enantiomer. As we advanced into unknown phospholipid territory, we found that we needed a mapping system to classify the new compounds in a non-machine-coded way and we needed a nomenclature that would put more weight on the nature of the chemical bonds between fatty acyl chains and the lipid backbone. Therefore, we adopted and expanded a modular nomenclature that had been introduced earlier by Szoka et al. ${ }^{18,19}$ The standard abbreviation for the headgroups (PC for phosphocholine) and fatty acyl chains (P for palmitoyl and $\mathrm{O}$ for oleoyl) were retained, and two letters would be used to unambiguously identify the chemical linker used to connect the fatty acyl chains to the backbone: "es" would be an ester; "et", an ether; "ad", an amide; and "ur", a urea. The standard POPC would therefore become Pes-Oes$\mathrm{PC}$, and its enantiomer, PC-Oes-Pes. The nomenclature allows us to understand how many unexplored lipid classes exist and how structurally limited nature essentially is. We were then able to move from natural phospholipids Xes-Xes-PC (X stands for any chain length and saturation/unsaturation) and Xet-Xet-PC into unknown lipids such as Xad-Xad-PC, Xad-
PC-Xad, and Xur-PC-Xur, which will all be covered in this feature article.

\section{PHOSPHOLIPID ASSEMBLIES AND WAYS TO STUDY THEM}

In nature, phospholipids are not found as individual molecules but rather are found in different types of ensembles. Because of the presence of two fatty acyl chains in one molecule, the phospholipids aggregate at very low concentrations. For DPPC (1, see Figure 2), the critical value lies at $0.46 \mathrm{nM}$ at $20{ }^{\circ} \mathrm{C} .{ }^{20}$ As a comparison, the single $\mathrm{C} 16$ chain lysoPC aggregates only at a 4 orders of magnitude higher concentration of $8.3 \mu \mathrm{M}^{20}$ The many possibilities in which phospholipids can associate lead to a broad spectrum of analytical methods that can be used for phospholipid characterization.

Typically, phospholipids in nature are found in bilayer arrangements. However, it is possible to study artificial monolayers of phospholipids. ${ }^{21}$ This has the big advantage of separating forces that are present in one membrane leaflet from forces present in a lipid bilayer. ${ }^{22}$ At an air/water interface, phospholipids spread into films that are one molecule thick. These films can be laterally compressed by a barrier, and the measured change in surface pressure certainly is one of the oldest ways to study ensembles of phospholipids. ${ }^{23}$ In the late 1980s and 1990s, powerful new techniques became available that enabled the probe-free (recently, a new push-pull pressure-sensitive fluorophore was introduced that does not change the organization of phospholipids, ${ }^{24}$ along with a multiparametric sensing fluorescent probe ${ }^{25}$ ) study of the deeper structure of monolayers using synchrotron X-ray diffraction, ${ }^{26,27}$ X-ray reflectivity, ${ }^{28}$ FTIR reflection absorption spectroscopy, ${ }^{29,30}$ specular reflections of neutrons, ${ }^{31}$ and Brewster angle microscopy. ${ }^{32,33}$ In particular, the X-ray scattering of phospholipid monolayers is now well understood, ${ }^{34}$ and beamlines such as the P08 of PETRA III of the Deutsches Synchrotron (DESY) allow routine measurements of lipid monolayers. In combination with the latest MatLab scripts, ${ }^{35}$ it is possible to analyze the results immediately after a measurement, which allows us to retake measurements make with faulty monolayer films. ${ }^{35}$

Various ways exist to formulate phospholipids into liposomes (or vesicles), uni- or multilamellar bilayers surrounding a water-filled core. ${ }^{36}$ Interestingly, for drug-delivery purposes drug molecules can be incorporated into the aqueous core or the membranes of liposomes. The drug should stay trapped 
inside the vesicle for a prolonged time. This depends on various factors, not the least important of which is the main phase-transition temperature of the bilayer. At and above this temperature, the phospholipid fatty acyl hydrocarbon chains have enough energy to change from an antiperiplanar (alltrans) conformation to a higher-energy conformation containing gauche interactions. ${ }^{37}$ This cooperative change in the phospholipid tails ${ }^{38}$ can be studied using differential scanning calorimetry and leads to a phase change from a gel phase to a liquid-crystalline phase, concomitant with the rapid release of the vesicle-entrapped cargo at and above the main phasetransition temperature. ${ }^{38,39}$

Unfortunately, the typical large unilamellar vesicles used for drug delivery are around $100 \mathrm{~nm}$ in diameter, so they are too small for optical characterization using even super-resolution microscopy. Here, cryogenic transition electron microscopy (cryo-TEM) helps us to obtain single snapshots of frozen vesicles. ${ }^{40}$ The geometrical factors determined from cryo-TEM images and size values measured with dynamic light scattering (DLS) can be used as starting values for fitting small-angle neutron scattering data. ${ }^{41,42}$ Together with small- and wideangle X-ray spectroscopy measurements, ${ }^{43}$ a statistically correct picture of the true vesicle population properties emerges.

\section{PHOSPHOLIPID SYNTHESIS}

The chemical space of artificial phospholipids is immense, but each new lipid obviously first needs to be created through chemical synthesis before any biophysical testing may begin. Unfortunately, synthesizing phospholipids is much harder than it looks on paper. ${ }^{44}$ Working with amphiphilic molecules requires special polar solvent mixtures, and standard laboratory techniques such as liquid/liquid extraction cannot be used. Fortunately, there is already a vast body of literature on natural and artificial phospholipid synthesis that would fill its own review article. ${ }^{6,14,45-52}$

Early lecithin synthesis in the 1920s was rather straightforward, and as part of the procedure, glycerol 1,2-distearate, phosphoric acid anhydride, and choline bicarbonate were vigorously kneaded for $4 \mathrm{~min}$ and the product was then extracted with almost-boiling benzene. ${ }^{44}$ Luckily, more sophisticated and less hands-on methods became available in the meantime. Nowadays, a handful of reagents exist that are useful for the synthesis of phospholipids, but unfortunately, many of them come at a prohibitively high cost because they are also used in polynucleotide synthesis. ${ }^{853}$ There is always a thin reactivity line between the (too) reactive $\mathrm{P}$ (III) reagents and the less-reactive $\mathrm{P}(\mathrm{V})$ reagents. The propensity of $\mathrm{P}(\mathrm{III})$ reagents to react twice with an alcohol is high, and unless special reagents such as benzyloxydichlorophosphine ${ }^{53}$ are used, the better option is usually $P(V)$ reagents if monosubstitution of the phosphate is desired. Over the years, 2-bromoethyl phosphorodichloridate (12), the Hirt reagent (Scheme 1), became a standard reagent for phospholipid synthesis. ${ }^{54}$ The Hirt reagent allows the chemist

Scheme 1. Synthesis of a Phosphocholine-Substituted Alcohol Using the Hirt Reagent, 2-Bromoethyl Phosphorodichloridate (12)

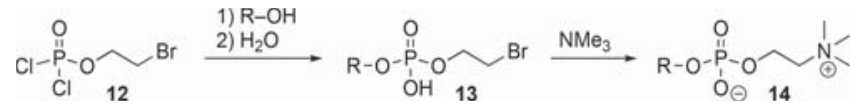

to introduce different headgroups such as the standard ethyl trimethylammonium group (14) or the ethyl dimethylammonium headgroups and more complex headgroups such as ethyl $N, N$-dimethyl- $N$-(2-dimethylaminoethyl) ammonium. ${ }^{55,56}$

Out of the vast open chemical space of artificial phospholipids we have chosen the genus of 1,3-diamidophospholipids as a synthesis and biophysical challenge. In hindsight, the choice turned out to be very lucky as this type of phospholipid indeed possesses many interesting features that will be discussed in this article.

A synthesis question arising early on is where to begin: at the head or introducing the tails later or vice versa? ${ }^{8}$ For the class of amido phospholipids, however, this question was easy to answer: at the headgroup. This is due to the fact that amido lipids without a phosphate headgroup are essentially insoluble. ${ }^{57}$ Intermolecular hydrogen bonding between amide groups leads to a $\beta$-sheet-like molecular arrangement. The importance of this hydrogen bonding effect showed up most impressively when the melting points of the bis-esther PesOH-Pes $\left(72-73{ }^{\circ} \mathrm{C}\right)$ was compared to the melting point of Pad-OH-Pad $\left(10,132-134{ }^{\circ} \mathrm{C}\right)$. Changing the molecular structure from an ester (hydrogen bond acceptor) to an amide (hydrogen bond donor and acceptor) led to an impressive stabilization of the compounds with this $60 \mathrm{~K}$ increase in the melting point. ${ }^{57}$ Synthesiswise it would be easy to add a phosphate to Pad-OH-Pad (10), but this is impossible because the reactant is insoluble. However, once a headgroup is present, the formation of intermolecular hydrogen bonds is hindered and the melting temperature again drops by $80 \mathrm{~K}^{57}$ Overall, this shows that the interface between the fatty acyl chains and the phosphate headgroup may have a large influence on the interlipid forces. We have used this concept when we have explored single-chain amphiphiles containing various chemical moieties such as amides, ureas, and thioureas. ${ }^{58}$ Interestingly, the thiourea moiety (9) leads to the buildup of a very stiff hydrogen bonding network between the interface regions of single-chain amphiphiles. This network was found to be self-healing and would reform itself after it was destroyed at the air-water interface. ${ }^{58}$

Interphospholipid hydrogen bonds lead to a stabilization of the vertical position between neighboring molecules. This has a positive effect on the polymerization behavior of the lipids. ${ }^{59}$ Typically, the diacetylene-substituted phospholipids can be polymerized only when the molecules are preorganized in a gel phase. ${ }^{13,14}$ Similarly, polyacetylene fatty acids preorganized at an air/water interface readily polymerize into two-dimensional carbon sheets. ${ }^{60}$ The system, however, is much more tolerable to vertical misalignment when the polymerizable chemical moieties are placed at the interface between the fatty acyl chains and the polar headgroup. ${ }^{59}$ Liposomes formulated from bis-acrylamido phospholipid (8) will easily polymerize into liposomal superstructures. ${ }^{59}$ The liposomes may also be admixed with nonpolymerizable lipids that can be washed away after polymerization, leaving behind holey structures.

\section{MONOLAYER PROPERTIES}

Our research on the biophysical properties of 1,3-diamidophospholipids started out at the air/water interface of a Langmuir/Pockels film balance (Figure 3) with both C16 PadPC-Pad (6) and its C18 homologue Sad-PC-Sad (7). Grazing incidence-angle X-ray diffraction (GIXD) would give a statistical average of the chain tilt angle and packing of the phospholipids with their headgroups pointing into the water 

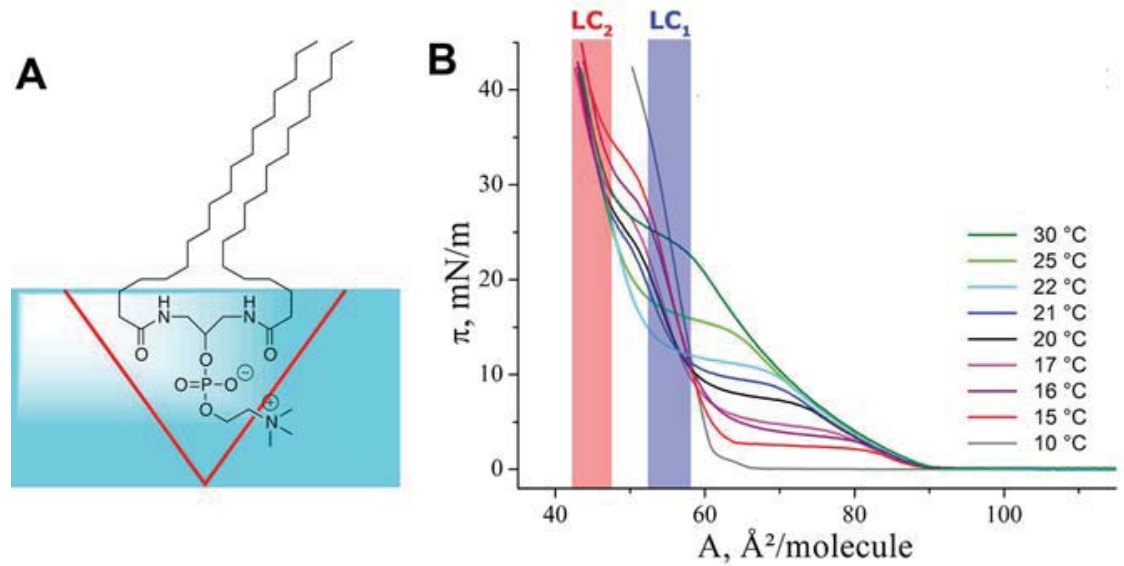

Figure 3. (A) Organization of a 1,3-diamidophospholipid at the air/water interface. The backbone forces the tails into a cone-shaped conformation. (B) Surface pressure/molecular area isotherms for Sur-PC-Sur (11) at various subphase temperatures. The two condensed phases are indicated. (A) Reprinted with permission from Fedotenko, L. A.; Stefaniu, C.; Brezesinski, G.; Zumbuehl, A. Monolayer Properties of 1,3Diamidophospholipids. Langmuir 2013, 29, 9428-9435. Copyright 2013 American Chemical Society. (B) Neuhaus, F.; Mueller, D.; Tanasescu, R.; Stefaniu, C.; Zaffalon, P. L.; Balog, S.; Ishikawa, T.; Reiter, R.; Brezesinski, G.; Zumbuehl, A. Against the Rules: Pressure Induced Transition from High to Reduced Order. Soft Matter 2018, 14, 3978-3986. Reproduced by permission of The Royal Society of Chemistry.

phase and their fatty acyl chains pointing into the air. GIXD signals are recorded only if there is indeed a global ordering of the chains. ${ }^{34,61,62}$ This ordering does depend on the temperature of the water subphase and on the compression of the monolayer film. For $35 \mathrm{mN} / \mathrm{m}$ and a $5{ }^{\circ} \mathrm{C}$ subphase temperature, the two chains of Sad-PC-Sad are tilted by $18^{\circ}$. Using a simple geometrical formula, the theoretical length of a C-C alkyl chain in its all antiperiplanar (all-trans) conformation can be calculated. ${ }^{63}$ For Sad-PC-Sad (7), this would be $21.6 \AA$, and the corresponding height of this chain tilted by $18^{\circ}$ would be $20.5 \AA$. However, the actual thickness of the lipid monolayer measured by X-ray reflectivity amounted to $19.2 \AA$, a clear indication that not the entire fatty acyl chain was in an all-antiperiplanar (all-trans) conformation and that the methylene groups near the glycerol backbone were disorganized (Figure 3). ${ }^{64}$ This disorganization at one end of the acyl chains has consequences for shorter-chain C16 PadPC-Pad (5): the overlap between the organized chains is not long enough and van der Waals interactions between the chains are not strong enough to keep the chains organized. The tails try to overcompensate for this with a larger tilt of $31.5^{\circ}$, but the tilt is not oriented to a preferred direction anymore and the chains are free to rotate. ${ }^{64}$ Such subtle changes in chain-chain interactions will become important in bilayers where Pad-PC-Pad (5) forms an interdigitated membrane but Sad-PC-Sad (7) mostly fails to form vesicles. ${ }^{22}$

On a side note, one effect is seen many times upon prolonged monolayer compression: when a Sad-PC-Sad (7) monolayer at $5{ }^{\circ} \mathrm{C}$ is kept for $2 \mathrm{~h}$ at a high lateral compression of $35 \mathrm{mN} / \mathrm{m}$, the tilt angle changes from 18 to $9^{\circ}$ probably because the intermolecular hydrogen bonds between the headgroups are broken, giving more rotational freedom to the chains and leading to a less-tilted state.

The power of the chemical synthesis of artificial phospholipids lies in the possibility to probe the limits of forces acting between molecules. Recently, we published the properties of the 1,3-diureaphospholipid Sur-PC-Sur (11, see Figure $3 \mathrm{~B}$ ), a molecule that contains two urea groups in the backbone and is capable of donating and accepting two hydrogen bonds per urea moiety. ${ }^{65}$ This lipid showed a profound change in the lateral chain area upon prolonged compression: The first liquid condensed phase $\left(\mathrm{LC}_{1}\right)$ was dominated by intermolecular hydrogen bonding, spacing the molecules widely apart and therefore forcing the tails into a large $52^{\circ}$ tilt. Upon further compression, the hydrogen bonds were broken, the molecules were organized into a less ordered, more densely packed condensed phase $\left(\mathrm{LC}_{2}\right)$, and the tails were again able to adopt a rotator phase with a much lower tilt angle of $35^{\circ}$. Interestingly, the phase diagram for a Sur-PC-Sur (11) monolayer shows two types of phase transitions, an exothermic change (from liquid expanded to condensed) and an endothermic change (between two condensed phases). This means that upon compression of the monolayer the film gets more disorganized, which is certainly counterintuitive. ${ }^{65}$

\section{BILAYER PROPERTIES}

The question of if a phospholipid bilayer is simply the sum of two monolayers is an interesting one. Ideally, a lipid monolayer at the air/water interface represents half of the combined forces in a bilayer membrane and typically both lipid monolayers and bilayers do have the same main phasetransition temperature, so the hypothesis is not far from reality. ${ }^{37}$ Of course any biophysical study on model phospholipids vastly underestimates the complexity of a natural membrane, but already such simpler models hint at additional forces being present in bilayers that are absent in monolayers. ${ }^{66}$ One such obvious case is found when the two leaflets of a bilayer membrane interdigitate. ${ }^{64,67,68}$

There is a tight correlation between the different parts of a phospholipid shown in Figure 1. Depending on the size of a phospholipid headgroup compared to its tails, which is the Israelachvili packing factor, phospholipids assemble into different superstructures when they are hydrated. ${ }^{69}$ Another equally important factor is the orientation of the phospholipid backbone in relation to the bilayer membrane normal. In a standard 1,2-diacylglycerolipid such as DPPC (1), the backbone is oriented parallel to the membrane normal. ${ }^{70}$ Exchanging the fatty acyl chains found in DPPC (1) for two 
fatty alkyl chains gives 1,2-di-O-hexadecyl-sn-glycero-3-phosphocholine (DHPC, 3) with a glycerol that is oriented perpendicularly to the bilayer normal. ${ }^{71,72}$ Similar to Sur-PCSur (11), where breaking intermolecular hydrogen bonds between phospholipid backbones leads to tighter packing, the absence of carbonyl groups in DHPC (3) backbones ${ }^{73}$ leads to a smaller area per hydrocarbon chain of $A_{x y}=22.8 \AA^{2}$ due to the closer chain packing. ${ }^{74}$ In diester phosphocholines, this closer chain packing would be penalized by a reduction of the freedom of the carbonyls to build up hydrogen bonds. ${ }^{75}$ The diether phospholipid reacts to the tighter packing by forming a fully interdigitated bilayer membrane ${ }^{75}$ in which one headgroup is essentially covering four alkyl chains. ${ }^{68}$ Recent improvements in computational chemistry showed that compared to DPPC (1), water penetrates deeper into DHPC (3) bilayers, leading to an increase in the headgroup size, and because of the better water organization, this leads to a decrease in water permeability, making ether lipids ideal for use in red blood cell and nerve myelin sheet membranes. ${ }^{76}$

Similar to DHPC (3), 1,3-diesterphospholipids (4) and 1,3diamidophospholipids (5) both have a backbone that is oriented perpendicularly to the bilayer normal. ${ }^{77,78}$ This means that the backbone dominates the Israelachvili packing factor and defines the phospholipid geometry by spacing the two acyl chains further apart compared to the spacing in a 1,2disubstituted phospholipid, leading to full bilayer membrane interdigitation. $^{78,79}$

That 1,3-diamidophospholipids form interdigitated membranes was apparent from early comparisons of monolayer and bilayer data. The 1,3-diamidophospholipids showed a bulk main phase-transition temperature that was linearly increasing with the length of the fatty acyl chains. ${ }^{64}$ However, the main transition temperature did not correlate with the analogous monolayer critical temperature at which a monolayer can no longer be compressed into a condensed state. The critical temperature for a 1,3-diamidophospholipid monolayer lies almost $10 \mathrm{~K}$ below the main transition of the bilayer. This shows that there are additional forces at play in a bilayer that are not present in monolayers. The most probable force stems from bilayer interdigitation. Additionally, the bilayer membrane interdigitation can be estimated from cryo-TEM images which for Rad-PC-Rad (6) has a very small value of $3.27 \mathrm{~nm} \pm$ $0.14 \mathrm{~nm} .{ }^{80}$ The interdigitation hypothesis was further confirmed by an electron density profile calculated from $\mathrm{X}$ ray data of gel-phase vesicles. ${ }^{79}$

Interdigitation may be a naturally occurring phenomenon when any biomembrane is stretched: the interdigitation stabilizes the membrane laterally. ${ }^{81}$ Apart from this stretching and the addition of small amphiphiles, ${ }^{82}$ interdigitation exists only for gel-phase phospholipids. Above the main phase transition, both 1,3-diesterphospholipids and 1,3-diamidophospholipids deinterdigitate. ${ }^{78,79}$ This process is reversible, and the membrane again interdigitates at temperatures below $T_{\mathrm{m}}$. The de-interdigitation can also be induced by adding as little as $10 \mathrm{~mol} \%$ cholesterol. $^{79}$ Cholesterol presumably fills the void space between the two fatty acyl chains of a 1,3-diamidophospholipid and so prevents the sliding-in of chains from the opposite membrane leaflet.

Membrane interdigitation firmly connects the two leaflets of a phospholipid bilayer. This leads to interesting phospholipid vesicle shapes, a field we have named vesicle origami.

\section{VESICLE ORIGAMI}

For simple geometric reasons, there are more phospholipid molecules in the outer membrane leaflet than in the inner membrane leaflet of a curved bilayer such as a vesicle. This means that the area of the inner membrane is smaller than the area of the outer membrane. Because the membrane cannot be stretched, this difference in areas induces a bending of the membrane into a curve. ${ }^{83}$

At room temperature, the two membrane leaflets of 1,3diamidophospholipid membranes are coupled by interdigitation. The bending energy of such a stiff membrane would be too high to form a closed vesicle; therefore, vesicles are formulated in a liquid-crystalline state above the main phasetransition temperature of the membrane. ${ }^{36,84}$ Upon rapid cooling to below $T_{m}$, the membranes reinterdigitate and faceted vesicles form. ${ }^{22}$ Basically, the geometry induced by a liquid-crystalline, deinterdigitated membrane has to be changed to a gel-phase interdigitated membrane: the membrane leaflets are connected, and the outer leaflet contains more lipids than the inner leaflet. This has to lead to faceted vesicles that contain membrane defects at the rim of the facets, where the outer membrane is continuous and the inner leaflet is broken (Figure 4). ${ }^{85}$ Similar faceted vesicles can be
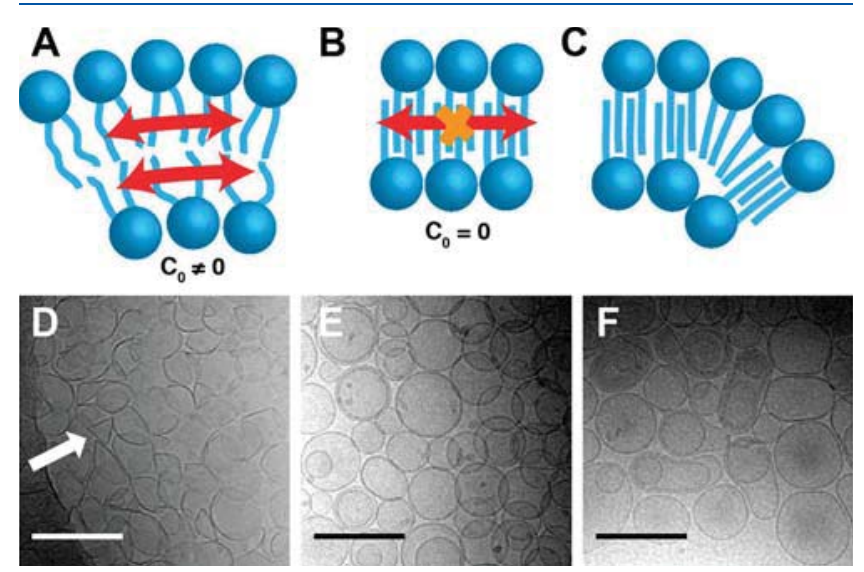

Figure 4. (A) Spherical vesicles with a positive curvature $C_{0}$ are formed from nonconnected liquid-crystalline bilayer membrane leaflets. (B) Interdigitated membranes are connected and must have membrane defects (C) when forced into closed 3D geometries. The lower row shows cryo-electron micrographs of extruded vesicles from (D) pure Pad-PC-Pad and Pad-PC-Pad with (E) 10 and (F) $40 \mathrm{~mol}$ $\%$ admixed cholesterol. The vesicles change from (D) rigid and faceted to (F) highly flexible. The scale bars are $200 \mathrm{~nm}$, and the white arrow points to an extremely faceted triangular vesicle. Reprinted with permission from Tanasescu, R.; Lanz, M. A.; Mueller, D.; Tassler, S.; Ishikawa, T.; Reiter, R.; Brezesinski, G.; Zumbuehl, A. Vesicle Origami and the Influence of Cholesterol on Lipid Packing. Langmuir 2016, 32, 4896-4903. Copyright 2016 American Chemical Society.

formulated from many different materials such as catanionic amphiphiles $^{86}$ and block copolymers. ${ }^{87}$ Essentially, the membrane is then made from a flexible rim and stiff facets, a multicomponent system that can adopt many geometries. ${ }^{88}$

Gel-phase phospholipid vesicles are faceted even when their membranes are not fully interdigitated. ${ }^{89}$ Therefore, the question arose as to if vesicle origami was also possible with a phospholipid membrane that is not fully interdigitated. We have resynthesized Pad-Pad-PC (2), a 1,2-diamidophospholipid that was initially intended to be an anti-AIDS drug. ${ }^{90}$ 
Similar to Sur-PC-Sur (11), Pad-Pad-PC (2) has strong intermolecular hydrogen bonds, but because of the 1,2compared to the 1,3-tail arrangement, the molecules can pack more tightly in the hydrogen-bond-defined condensed state. ${ }^{35}$ In fact, extremely tight subgel herringbone packing is found, which leads to a very low area per lipid tail of $19 \AA^{2}$. The intramolecular hydrogen bonding is so strong that a wideangle X-ray scattering signal appears akin to the $4.75 \AA$ repeat distance seen between hydrogen-bonded peptide $\beta$-sheets. ${ }^{35,91}$ Pad-Pad-PC (2) forms an extremely stiff bilayer and is forced into a $3 \mathrm{D}$ assembly, where the vesicle maximizes its flat surfaces and minimizes its defect lines; the geometrical form satisfying these constraints is a cube. ${ }^{35}$ Using vesicle origami, we managed to formulate the first free-standing, nontemplated soft matter cuboid shortly after the first DNA origamitemplated vesicle cube was reported. ${ }^{92}$

\section{MECHANORESPONSIVE DRUG DELIVERY}

Modern nanomedicine is currently suffering from a lack of translation from in vitro experiments via in animal experiments to in human medical applications. The translation of in vitro work to in vivo work remains challenging and unpredictable. ${ }^{93}$ A recent review has unmistakably shown that tumor targeting does not live up to its expectations in humans. ${ }^{94}$ Long-standing facts such as passive targeting via the enhanced permeation and retention (EPR) effect turned out to be more heterogeneous in humans than in optimized fast-growing xenografted mice tumor models with dense vasculature. ${ }^{95}$ It has become clear that once in contact with blood, a liposome is coated with a dynamic corona, which changes the "biological identity" of the liposome such as its size and aggregation state. ${ }^{96}$ Additionally, the interaction between ions and headgroups can lead to significant physical changes on the liposomes, such as vesicle fusion. ${ }^{97}$ Alternative, clever methods are being explored and are entering clinical trials, such as the use of red blood cells as drug delivery devices. ${ }^{98}$ At the same time, our understanding grows as to how drug delivery actually works and how the components of the blood, like leukocytes, may interfere with the planned process. ${ }^{99}$

One decade ago, we began working on a collaborative project in what is now called mechanoresponsive drug delivery. ${ }^{100,101}$ Seeing the lack of efficiency of biological and chemical drug targeting, we asked ourselves if it would be possible to use physics, more specifically, shear forces to disrupt bilayer vesicle membranes and to use such vesicles as an alternative form of targeted drug delivery (Figure 5). ${ }^{102}$
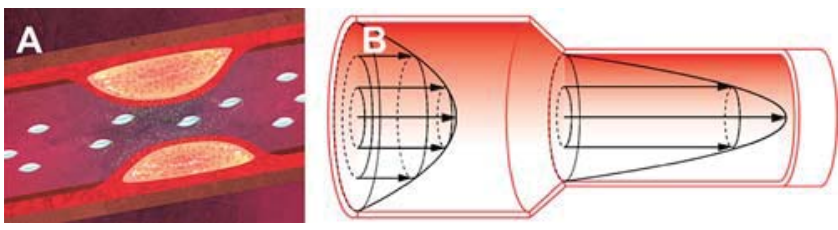

Figure 5. (A) Schematic depiction of the concept of mechanoresponsive drug delivery: special responsive vesicles release their cargo upon passing through a region of elevated shear forces, such as a constricted human artery. (B) Schematic depiction of the velocity profile of blood flowing through idealized cylindrically shaped vessels. At a constriction, the blood velocity and therefore also the wall shear stress increase. ${ }^{102}$ Saxer, T.; Zumbuehl, A.; Müller, B. The Use of Shear Stress for Targeted Drug Delivery. Cardiovasc. Res. 2013, 99, 328-333, used by permission of the European Society of Cardiology.
The standard vesicles used for the delivery of hydrophobic and hydrophilic drugs ${ }^{103}$ are in a liquid-crystalline phase; therefore, they are in an energy-minimized spherical conformation. ${ }^{104}$ Despite being self-assembled from individual amphiphiles, vesicles are surprisingly resistant to lateral membrane disruption. Calculations based on research reported by Bernard et al. show that $100 \mathrm{~nm}$ liposomes formulated from natural lipids and surfactants require shear stresses above $40 \mathrm{~Pa}$ to disrupt their membranes. ${ }^{105}$ The wall shear stress in a normal artery varies between 0.5 and $1.5 \mathrm{~Pa}$ and increases significantly by at least 1 order of magnitude at an arterial constriction. ${ }^{106}$ This has the consequence that normal liquidcrystalline vesicles cannot be used for mechanoresponsive drug delivery. Gel-phase vesicles such as those made from Pad-PCPad (5) and Rad-PC-Rad (6), on the other hand, are suited for mechanoresponsive drug delivery. Our initial experiments included vortex shaking of vesicles filled with 5(6)-carboxyfluorescein. The liposomes were shaken on a vortex shaker at $2500 \mathrm{rpm}$ for several seconds at different temperatures (Figure 6). Mechanoresponsive cargo release can work only when the vesicles are in the gel phase. Therefore, at a simulated body temperature of $37{ }^{\circ} \mathrm{C}$, Pad-PC-Pad vesicles $\left(5, T_{\mathrm{m}}=37{ }^{\circ} \mathrm{C}\right)$ fully release their cargo (Figure 6, right), whereas Rad-PC-Rad vesicles $\left(6, T_{\mathrm{m}}=44.7^{\circ} \mathrm{C}\right)$ and DPPC vesicles $\left(1, T_{\mathrm{m}}=42^{\circ} \mathrm{C}\right)$ remain active. The DPPC (1) vesicles, however, show only an initial burst release and then remain tight for the rest of the experiment. $^{107}$

To translate this technology into clinics, an important question to answer is whether the 1,3-diamidophospholipids are toxic. The systemic administration of liposomes may trigger a dangerous pseudoallergic reaction: an immediate activation of the patient's innate immune system. ${ }^{108}$ Surprisingly, our initial screening in pigs showed only very mild activation of the alternative pathway of the complement, a reaction also seen with approved natural phospholipid liposome formulations. ${ }^{80}$ The low toxicity was also induced by the nonspherical shape: Moghimi et al. have recently shown that spherically shaped liposomes promote cardiopulmonary stress in pigs, and this effect can be mitigated by changing the shape to rods or disks. ${ }^{109}$ The lack of toxicity is a promising factor for moving the project forward. ${ }^{110,111}$

Interestingly, the concept of shear-sensitive drug delivery has emerged at the same time in two different laboratories divided by the Atlantic Ocean, with Ingber et al. disclosing their approach using drug-impregnated nanoparticle aggregates. ${ }^{112}$ In past years, more groups started to work on mechanoresponsive drug delivery. ${ }^{101}$ Grinstaff et al. have defined the field as harnessing "existing physiological and/or externally applied forces to provide spatiotemporal control over the release of active agents". ${ }^{101}$ The field is open for drug delivery systems based on mechanical forces of compression, tension, and shear ${ }^{101}$ and represents an attractive alternative to the classical drug delivery routes. The most recent example of a shearsensitive liposomal system points to the bright future of an exciting field of research. ${ }^{113}$

\section{SUMMARY}

The chemical space of artificial phospholipids is vast, and most molecules are awaiting their synthesis. In this article, only a handful of new molecules were described. Nonetheless, the biophysical properties of this limited choice were already surprisingly rich. Soft matter phospholipid assemblies turned out to be tunable and to adapt their shapes in reaction to 

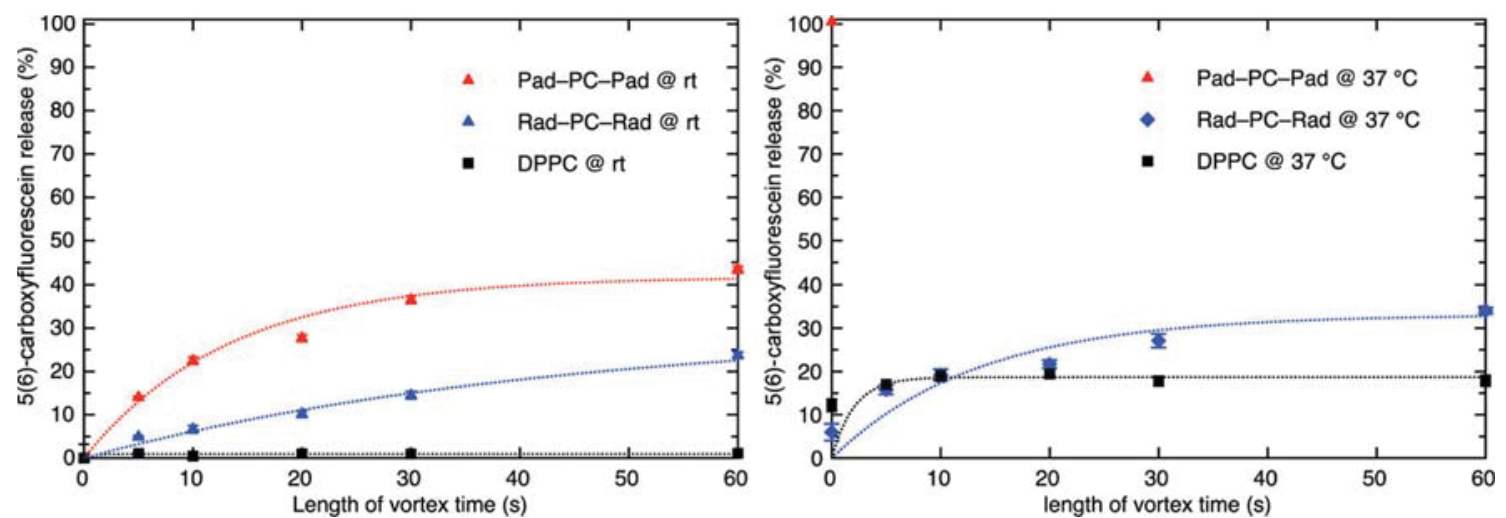

Figure 6. Vortex release experiments at 2500 rpm with 5(6)-carboxyfluorescein-filled vesicles formulated from different pure phospholipids. PadPC-Pad vesicles with $T_{\mathrm{m}}=37^{\circ} \mathrm{C}$ are becoming leaky at simulated body temperature. The other two formulations with $T_{\mathrm{m}}>37^{\circ} \mathrm{C}$ stay in the gel phase and show shaking-dependent release kinetics. DPPC vesicles release their cargo only at the very beginning of the shaking period. Reprinted with permission from Neuhaus, F.; Mueller, D.; Tanasescu, R.; Balog, S.; Ishikawa, T.; Brezesinski, G.; Zumbuehl, A. Synthesis and Biophysical Characterization of an Odd-Numbered 1,3-Diamidophospholipid. Langmuir 2018, 34, 3215-3220. Copyright 2018 American Chemical Society.

mechanical stress, ${ }^{100}$ temperature change, ${ }^{22}$ and the addition of cholesterol. ${ }^{79}$ Chemical synthesis of phospholipids is key to exploring the frontiers of vesicle origami and going beyond into applications of the concept in future personalized medicine.

\section{AUTHOR INFORMATION}

\section{Corresponding Author}

*E-mail: andreas.zumbuehl@unifr.ch.

\section{ORCID}

Andreas Zumbuehl: 0000-0002-8003-4432

Notes

The author declares no competing financial interest.

\section{ACKNOWLEDGMENTS}

My sincere thanks goes to all of the various researchers and funding agencies, such as the Swiss National Science Foundation and the National Center of Competence in
Research in Chemical Biology, that helped me on this journey into the world of artificial phospholipids. I especially thank my long-term collaborators and friends Prof. Gerald Brezesinski and Prof. Bert Müller.

\section{REFERENCES}

(1) Mouritsen, O. G. Life: As a Matter of Fat; Springer: Berlin, 2005.

(2) Singer, S. J.; Nicolson, G. L. The fluid mosaic model of the structure of cell membranes. Science 1972, 175, 720-731.

(3) Harayama, T.; Riezman, H. Understanding the diversity of membrane lipid composition. Nat. Rev. Mol. Cell Biol. 2018, 19, 281296.

(4) Hannich, J. T.; Mellal, D.; Feng, S. H.; Zumbuehl, A.; Riezman, $\mathrm{H}$. Structure and conserved function of iso-branched sphingoid bases from the nematode Caenorhabditis elegans. Chem. Sci. 2017, 8, 3676-3686.

(5) Sharp, P. A.; Cooney, C. L.; Lees, J.; Sasisekharan, R.; Yaffe, M. B.; Bhatia, S. N.; Jacks, T. E.; Lauffenburger, D. A.; Langer, R.; Hammond, P. T.; Sur, M. The Convergence of the Life Sciences, Physical Sciences, and Engineering; Massachusetts Institute of Technology: Cambridge, MA, 2011.

(6) Bhattacharya, S.; Biswas, J. Understanding Membranes through the Molecular Design of Lipids. Langmuir 2010, 26, 4642-4654.

(7) Zumbuehl, A. Nonnatural Phospholipids: Probing Nature's Modular Platform. Chimia 2009, 63, 63-65.

(8) Fedotenko, I. A.; Holme, M. N.; Tanasescu, R.; Zaffalon, P. L.; Zumbuehl, A. Putting the 'P' into Phospholipids. Chimia 2011, 65, 859-862.

(9) Bonsen, P. P. M.; Burbach-Westerhuis, G. J.; de Haas, G. H.; van Deenen, L. L. M. Chemical Synthesis of some Lecithin Analogues Potential Inhibitors of Phospholipase A. Chem. Phys. Lipids 1972, 8, 199-220.

(10) Ernst, R.; Ejsing, C. S.; Antonny, B. Homeoviscous Adaptation and the Regulation of Membrane Lipids. J. Mol. Biol. 2016, 428, 4776-4791.

(11) Andresen, T. L.; Davidsen, J.; Begtrup, M.; Mouritsen, O. G.; Jørgensen, K. Enzymatic Release of Antitumor Ether Lipids by Specific Phospholipase A2 Activation of Liposome-Forming Prodrugs. J. Med. Chem. 2004, 47, 1694-1703.

(12) Byun, H.-S.; Bittman, R. Efficient Stereospecific Synthesis of Diamide Analogs of Phosphatidylcholine Starting from 1-(4'Methoxyphenyl)-sn-glycerol. J. Org. Chem. 1996, 61, 8706-8708.

(13) Hub, H. H.; Hupfer, B.; Koch, H.; Ringsdorf, H. Polyreactions in Ordered Systems 0.20. Polymerizable Phospholipid Analogs - New Stable Biomembrane and Cell Models. Angew. Chem., Int. Ed. Engl. 1980, 19, 938-940. 
(14) Johnston, D. S.; Sanghera, S.; Manjonrubio, A.; Chapman, D. The Formation of Polymeric Model Biomembranes from Diacetylenic Fatty-Acids and Phospholipids. Biochim. Biophys. Acta, Biomembr. 1980, 602, 213-216.

(15) Wenk, M. R. The emerging field of lipidomics. Nat. Rev. Drug Discovery 2005, 4, 594-610.

(16) Quehenberger, O.; Armando, A. M.; Brown, A. H.; Milne, S. B.; Myers, D. S.; Merrill, A. H.; Bandyopadhyay, S.; Jones, K. N.; Kelly, S.; Shaner, R. L.; Sullards, C. M.; Wang, E.; Murphy, R. C.; Barkley, R. M.; Leiker, T. J.; Raetz, C. R. H.; Guan, Z.; Laird, G. M.; Six, D. a.; Russell, D. W.; McDonald, J. G.; Subramaniam, S.; Fahy, E.; Dennis, E. a. Lipidomics reveals a remarkable diversity of lipids in human plasma. J. Lipid Res. 2010, 51, 3299-3305.

(17) IUPAC-IUB Commission on Biochemical Nomenclature. The nomenclature of lipids. Lipids 1977, 12, 455-468.

(18) Huang, Z.; Szoka, F. C. Sterol-Modified Phospholipids: Cholesterol and Phospholipid Chimeras with Improved Biomembrane Properties. J. Am. Chem. Soc. 2008, 130, 15702-15712.

(19) Fedotenko, I. A.; Zaffalon, P. L.; Favarger, F.; Zumbuehl, A. The synthesis of 1,3-diamidophospholipids. Tetrahedron Lett. 2010 51, 5382-5384.

(20) Marsh, D. Handbook of Lipid Bilayers, 2nd ed.; CRC Press: Boca Raton, FL, 2013.

(21) Moehwald, H.; Brezesinski, G. From Langmuir Monolayers to Multilayer Films. Langmuir 2016, 32, 10445-10458.

(22) Weinberger, A.; Tanasescu, R.; Stefaniu, C.; Fedotenko, I. A.; Favarger, F.; Ishikawa, T.; Brezesinski, G.; Marques, C. M.; Zumbuehl, A. Bilayer Properties of 1,3-Diamidophospholipids. Langmuir 2015, 31, 1879-1884.

(23) Heimburg, T. Thermal Biophysics of Membranes; Wiley-VCH: Weinheim, Germany, 2007.

(24) Neuhaus, F.; Zobi, F.; Brezesinski, G.; Dal Molin, M.; Matile, S.; Zumbuehl, A. Correlation of surface pressure and hue of planarizable push-pull chromophores at the air/water interface. Beilstein J. Org. Chem. 2017, 13, 1099-1105.

(25) Gulyani, A.; Dey, N.; Bhattacharya, S., Highly responsive fluorescent assemblies allow unique, multiparametric sensing of phospholipid membrane environment. Chemistry 2018 , DOI: $10.1002 /$ chem.201803627.

(26) Kjaer, K.; Als-Nielsen, J.; Helm, C. A.; Laxhuber, L. A.; Möhwald, H. Ordering in Lipid Monolayers Studied by Synchrotron X-Ray Diffraction and Fluorescence Microscopy. Phys. Rev. Lett. 1987, 58, 2224-2227.

(27) Dutta, P.; Peng, J. B.; Lin, B.; Ketterson, J. B.; Prakash, M.; Georgopoulos, P.; Ehrlich, S. X-Ray-diffraction studies of organic monolayers on the surface of water. Phys. Rev. Lett. 1987, 58, 2228.

(28) Helm, C. A.; Mohwald, H.; Kjaer, K.; Alsnielsen, J. Phospholipid Monolayer Density Distribution Perpendicular to the Water-Surface - a Synchrotron X-Ray Reflectivity Study. Europhys. Lett. 1987, 4, 697-703.

(29) Dluhy, R. A.; Mendelsohn, R. Emerging techniques in biophysical FT-IR. Anal. Chem. 1988, 60, 269A.

(30) Grandbois, M.; Desbat, B.; Blaudez, D.; Salesse, C. Polarization-Modulated Infrared Reflection Absorption Spectroscopy Measurement of Phospholipid Monolayer Hydrolysis by Phospholipase C. Langmuir 1999, 15, 6594-6597.

(31) Penfold, J.; Thomas, R. K. The application of the specular reflection of Neutrons to the study of surfaces and interfaces. J. Phys.: Condens. Matter 1990, 2, 1369.

(32) Honig, D.; Mobius, D. Direct Visualization of Monolayers at the Air-Water-Interface by Brewster-Angle Microscopy. J. Phys. Chem. 1991, 95, 4590-4592.

(33) Henon, S.; Meunier, J. Microscope at the Brewster-Angle Direct observation of 1st-order phase-transitions in monolayers. Rev. Sci. Instrum. 1991, 62, 936.

(34) Kaganer, V. M.; Möhwald, H.; Dutta, P. Structure and phase transitions in Langmuir monolayers. Rev. Mod. Phys. 1999, 71, 779819.
(35) Neuhaus, F.; Mueller, D.; Tanasescu, R.; Balog, S.; Ishikawa, T.; Brezesinski, G.; Zumbuehl, A. Vesicle Origami: Cuboid Phospholipid Vesicles Formed by Template-Free Self-Assembly. Angew. Chem., Int. Ed. 2017, 56, 6515-6518.

(36) Walde, P. Preparation of vesicles (liposomes). Encyclopedia of Nanoscience and Nanotechnology 2004, 9, 37.

(37) Nagle, J. F. Theory of the Main Lipid Bilayer Phase-Transition. Annu. Rev. Phys. Chem. 1980, 31, 157-195.

(38) Blicher, A.; Wodzinska, K.; Fidorra, M.; Winterhalter, M.; Heimburg, T. The temperature dependence of lipid membrane permeability, its quantized nature, and the influence of anesthetics. Biophys. J. 2009, 96, 4581-4591.

(39) Mellal, D.; Zumbuehl, A. Exit-strategies - smart ways to release phospholipid vesicle cargo. J. Mater. Chem. B 2014, 2, 247-252.

(40) Kuntsche, J.; Horst, J. C.; Bunjes, H. Cryogenic transmission electron microscopy (cryo-TEM) for studying the morphology of colloidal drug delivery systems. Int. J. Pharm. 2011, 417, 120-137.

(41) Bressler, I.; Kohlbrecher, J.; Thunemann, A. F. SASfit: a tool for small-angle scattering data analysis using a library of analytical expressions. J. Appl. Crystallogr. 2015, 48, 1587-1598.

(42) Kohlbrecher, J.; Studer, A. Transformation cycle between the spherically symmetric correlation function, projected correlation function and differential cross section as implemented in SASfit. J. Appl. Crystallogr. 2017, 50, 1395-1403.

(43) Glatter, O.; Kratky, O. Small Angle X-ray Scattering; Academic Press: London, 1982; p 267.

(44) Grün, A.; Limpächer, R. Synthese der Lecithine. Ber. Dtsch. Chem. Ges. B 1926, 59, 1350-1360.

(45) Eibl, H. Phospholipid Synthesis - Oxazaphospholanes and Dioxaphospholanes as Intermediates. Proc. Natl. Acad. Sci. U. S. A. 1978, 75, 4074-4077.

(46) Buldt, G.; Gally, H. U.; Seelig, A.; Seelig, J.; Zaccai, G. NeutronDiffraction Studies on Selectively Deuterated Phospholipid Bilayers. Nature 1978, 271, 182-184.

(47) Eibl, H. Synthesis of Glycerophospholipids. Chem. Phys. Lipids 1980, 26, 405-429.

(48) Ringsdorf, H.; Schlarb, B.; Venzmer, J. Molecular Architecture and Function of Polymeric Oriented Systems - Models for the Study of Organization, Surface Recognition, and Dynamics of Biomembranes. Angew. Chem., Int. Ed. Engl. 1988, 27, 113-158.

(49) Ravily, V.; Santaella, C.; Vierling, P.; Gulik, a. Phase behavior of fluorocarbon di-O-alkyl-glycerophosphocholines and glycerophosphoethanolamines and long-term shelf stability of fluorinated liposomes. Biochim. Biophys. Acta, Biomembr. 1997, 1324, 1-17.

(50) Bhattacharya, S.; Subramanian, M. Synthesis of novel phosphatidylcholine lipids with fatty acid chains bearing aromatic units. Generation of oxidatively stable, fluid phospholipid membranes. Tetrahedron Lett. 2002, 43, 4203-4206.

(51) Meister, A.; Blume, A. Self-assembly of bipolar amphiphiles. Curr. Opin. Colloid Interface Sci. 2007, 12, 138-147.

(52) Lewis, R. N. A. H.; McElhaney, R. N., The Mesomorphic Phase Behavior of Lipid Bilayers. In The Structure of Biological Membranes; Yeagle, P. L., Ed.; CRC Press: Boca Raton, FL, 2012; Vol. 1, pp 1990.

(53) Zaffalon, P. L.; Zumbuehl, A. BODP - A Versatile Reagent for Phospholipid Synthesis. Synthesis 2011, 2011, 778-782.

(54) Hirt, R.; Berchtold, R. Zur Synthese der Phosphatide. Eine neue Synthese der Lecithine. Pharm. Acta Helv. 1958, 33, 349-356.

(55) Drescher, S.; Meister, A.; Graf, G.; Hause, G.; Blume, A.; Dobner, B. General synthesis and aggregation behaviour of new single-chain bolaphospholipids: Variations in chain and headgroup structures. Chem. - Eur. J. 2008, 14, 6796-6804.

(56) Blume, A.; Drescher, S.; Graf, G.; Köhler, K.; Meister, A. Selfassembly of different single-chain bolaphospholipids and their miscibility with phospholipids or classical amphiphiles. Adv. Colloid Interface Sci. 2014, 208, 264-278.

(57) Zaffalon, P. L.; D’Anna, V.; Hagemann, H.; Zumbuehl, A. Study of surfactant alcohols with various chemical moieties at the hydrophilic-hydrophobic interface. RSC $A d v$. 2013, 3, 7237-7244. 
(58) Stefaniu, C.; Zaffalon, P. L.; Carmine, A.; Verolet, Q.; Fernandez, S.; Wesolowski, T. A.; Brezesinski, G.; Zumbuehl, A. Rigid Urea and Self-Healing Thiourea Ethanlamine Monolayers. Langmuir 2015, 31, 1296-1302.

(59) Zaffalon, P.-L.; Stalder, E.; Fedotenko, I. a.; Favarger, F.; Zumbuehl, A. The synthesis of an amine-bearing polymerizable phospholipid. Tetrahedron Lett. 2011, 52, 4215-4217.

(60) Schrettl, S.; Stefaniu, C.; Schwieger, C.; Pasche, G.; Oveisi, E.; Fontana, Y.; Morral, A. F. I.; Reguera, J.; Petraglia, R.; Corminboeuf, C.; Brezesinski, G.; Frauenrath, H. Functional carbon nanosheets prepared from hexayne amphiphile monolayers at room temperature. Nat. Chem. 2014, 6, 468-476.

(61) Giner-Casares, J. J.; Brezesinski, G.; Möhwald, H. Langmuir monolayers as unique physical models. Curr. Opin. Colloid Interface Sci. 2014, 19, 176-182.

(62) Stefaniu, C.; Brezesinski, G. X-ray investigation of monolayers formed at the soft air/water interface. Curr. Opin. Colloid Interface Sci. 2014, 19, 216-227.

(63) Kitaigorodskii, A. I. Organic Chemical Crystallography; Consultants Bureau: New York, 1961.

(64) Fedotenko, L. A.; Stefaniu, C.; Brezesinski, G.; Zumbuehl, A. Monolayer properties of 1,3-diamidophospholipids. Langmuir 2013, $29,9428-9435$.

(65) Neuhaus, F.; Mueller, D.; Tanasescu, R.; Stefaniu, C.; Zaffalon, P. L.; Balog, S.; Ishikawa, T.; Reiter, R.; Brezesinski, G.; Zumbuehl, A. Against the rules: pressure induced transition from high to reduced order. Soft Matter 2018, 14, 3978-3986.

(66) Schick, M. Strongly Correlated Rafts in Both Leaves of an Asymmetric Bilayer. J. Phys. Chem. B 2018, 122, 3251-3258.

(67) Slater, J. L.; Huang, C.-H. Interdigitated Bilayer Membranes. Prog. Lipid Res. 1988, 27, 325-359.

(68) Slater, J. L.; Huang, C.-H. Lipid Bilayer Interdigitation. The Structure of Biological Membranes, 2nd ed.; CRC Press: 2005; pp 121145

(69) Israelachvili, J. N. Intermolecular and Surface Forces, 3rd ed.; Academic Press: San Diego, 2011.

(70) Büldt, G.; Wohlgemuth, R. The Headgroup Conformation of Phospholipids in Membranes. J. Membr. Biol. 1981, 58, 81-100.

(71) Rattay, B.; Brezesinski, G.; Dobner, B.; Förster, G.; Nuhn, P. Influence of $\alpha$-branched fatty acid chains on the thermotropic behaviour of racemic 1-O-hexadecyl-2-acyl-glycero-3- phosphocholines. Chem. Phys. Lipids 1995, 75, 81-91.

(72) Mattjus, P.; Bittman, R.; Slotte, J. P. Molecular interaction and lateral domain formation in monolayers containing cholesterol and phosphatidylcholines with acyl- or alkyl-linked C16 chains. Langmuir 1996, 12, 1284-1290.

(73) Ruocco, M. J.; Siminovitch, D. J.; Griffin, R. G. Comparative Study of the Gel Phases of Ether- and Ester-Linked Phosphatidylcholines. Biochemistry 1985, 24, 2406-2411.

(74) Brezesinski, G.; Dietrich, A.; Struth, B.; Böhm, C.; Bouwman, W. G.; Kjaer, K.; Möhwald, H. Influence of ether linkages on the structure of double-chain phospholipid monolayers. Chem. Phys. Lipids 1995, 76, 145-157.

(75) Laggner, P.; Lohner, K.; Degovics, G.; Müller, K.; Schuster, A. Structure and thermodynamics of the Dihexadecylphosphatidylcholine-water system. Chem. Phys. Lipids 1987, 44, 31-60.

(76) Leonard, A. N.; Pastor, R. W.; Klauda, J. B. Parameterization of the CHARMM All-Atom Force Field for Ether Lipids and Model Linear Ethers. J. Phys. Chem. B 2018, 122, 6744-6754.

(77) Buldt, G.; Dehaas, G. H. Conformational Differences between sn-3-Phospholipids and sn-2-Phospholipids - a Neutron and X-RayDiffraction Investigation. J. Mol. Biol. 1982, 158, 55-71.

(78) Serrallach, E. N.; Dijkman, R.; de Haas, G. H.; Shipley, G. G. Structure and Thermotropic Properties of 1,3-Dipalmitoyl-glycero-2 phosphocholine. J. Mol. Biol. 1983, 170, 155-174.

(79) Tanasescu, R.; Lanz, M. A.; Mueller, D.; Tassler, S.; Ishikawa, T.; Reiter, R.; Brezesinski, G.; Zumbuehl, A. Vesicle Origami and the Influence of Cholesterol on Lipid Packing. Langmuir 2016, 32, 48964903.
(80) Matviykiv, S.; Buscema, M.; Gerganova, G.; Meszaros, T.; Kozma, G. T.; Mettal, U.; Neuhaus, F.; Ishikawa, T.; Szebeni, J.; Zumbuehl, A.; Müller, B. Immunocompatibility of Rad-PC-Rad liposomes in vitro, base on human complement activation and cytokine release. Precision Nanomedicine 2018, 1, 45-67.

(81) Shigematsu, T.; Koshiyama, K.; Wada, S. Stretch-Induced Interdigitation of a Phospholipid/Cholesterol Bilayer. J. Phys. Chem. B 2018, 122, 2556-2563.

(82) Kamal, M. A.; Raghunathan, V. A. Naphthalene Derivatives Induce Acyl Chain Interdigitation in Dipalmitoylphosphatidylcholine Bilayers. J. Phys. Chem. B 2016, 120, 164-172.

(83) Zimmerberg, J.; Kozlov, M. M. How proteins produce cellular membrane curvature. Nat. Rev. Mol. Cell Biol. 2006, 7, 9-19.

(84) Antonietti, M.; Förster, S. Vesicles and Liposomes: A SelfAssembly Principle Beyond Lipids. Adv. Mater. 2003, 15, 1323-1333.

(85) Noguchi, H. Polyhedral vesicles: a Brownian dynamics simulation. Phys. Rev. E: Stat. Phys., Plasmas, Fluids, Relat. Interdiscip. Top. 2003, 67, 041901.

(86) Dubois, M.; Demé, B.; Gulik-Krzywicki, T.; Dedieu, J. C.; Vautrin, C.; Désert, S.; Perez, E.; Zemb, T. Self-assembly of regular hollow icosahedra in salt-free catanionic solutions. Nature 2001, 411 , 672-675.

(87) Deng, Y.; Ling, J.; Li, M. H. Physical stimuli-responsive liposomes and polymersomes as drug delivery vehicles based on phase transitions in the membrane. Nanoscale 2018, 10, 6781-6800.

(88) Vernizzi, G.; Sknepnek, R.; de la Cruz, M. O. Platonic and Archimedean geometries in multicomponent elastic membranes. Proc. Natl. Acad. Sci. U. S. A. 2011, 108, 4292-4296.

(89) Sieber, S.; Grossen, P.; Detampel, P.; Siegfried, S.; Witzigmann, D.; Huwyler, J. Zebrafish as an early stage screening tool to study the systemic circulation of nanoparticulate drug delivery systems in vivo. J. Controlled Release 2017, 264, 180-191.

(90) Jia, C.; Haines, A. H. Diamide analogues of phosphatidyl choline as potential anti-AIDS agents. J. Chem. Soc., Perkin Trans. 1 1993, 2521-2523.

(91) Chevalier, N. R.; Chevallard, C.; Goldmann, M.; Brezesinski, G.; Guenoun, P. $\mathrm{CaCO}_{3}$ Mineralization under $\beta$-Sheet Forming Peptide Monolayers. Cryst. Growth Des. 2012, 12, 2299-2305.

(92) Dong, Y.; Yang, Y. R.; Zhang, Y.; Wang, D.; Wei, X.; Banerjee, S.; Liu, Y.; Yang, Z.; Yan, H.; Liu, D. Cuboid Vesicles Formed by Frame-Guided Assembly on DNA Origami Scaffolds. Angew. Chem., Int. Ed. 2017, 56, 1586-1589.

(93) Østrem, R. G.; Parhamifar, L.; Pourhassan, H.; Clergeaud, G.; Nielsen, O. L.; Kjær, A.; Hansen, A. E.; Andresen, T. L. Secretory phospholipase $\mathrm{A}_{2}$ responsive liposomes exhibit a potent antineoplastic effect in vitro, but induce unforeseen severe toxicity in vivo. J. Controlled Release 2017, 262 (Supplement C), 212-221.

(94) Wilhelm, S.; Tavares, A. J.; Dai, Q.; Ohta, S.; Audet, J.; Dvorak, H. F.; Chan, W. C. W. Analysis of nanoparticle delivery to tumours. Nat. Rev. Mater. 2016, 1, 16014.

(95) Rosenblum, D.; Joshi, N.; Tao, W.; Karp, J. M.; Peer, D. Progress and challenges towards targeted delivery of cancer therapeutics. Nat. Commun. 2018, 9, 1410.

(96) Caracciolo, G.; Palchetti, S.; Digiacomo, L.; Chiozzi, R. Z. Z.; Capriotti, A. L.; Amenitsch, H.; Tentori, P. M.; Palmieri, V.; Papi, M.; Cardarelli, F.; Pozzi, D.; Lagana, A. Human Biomolecular Corona of Liposomal Doxorubicin: The Overlooked Factor in Anticancer Drug Delivery. ACS Appl. Mater. Interfaces 2018, 10, 22951-22962.

(97) Liu, Y.; Liu, J. Cu2+-Directed Liposome Membrane Fusion, Positive-Stain Electron Microscopy, and Oxidation. Langmuir 2018, 34, 7545-7553.

(98) Han, X.; Wang, C.; Liu, Z. Red Blood Cells as Smart Delivery Systems. Bioconjugate Chem. 2018, 29, 852-860.

(99) Betker, J. L.; Jones, D.; Childs, C. R.; Helm, K. M.; Terrell, K.; Nagel, M. A.; Anchordoquy, T. J. Nanoparticle uptake by circulating leukocytes: A major barrier to tumor delivery. J. Controlled Release 2018, 286, 85-93.

(100) Holme, M. N.; Fedotenko, I. A.; Abegg, D.; Althaus, J.; Babel, L.; Favarger, F.; Reiter, R.; Tanasescu, R.; Zaffalon, P.-L.; Ziegler, A.; 
Müller, B.; Saxer, T.; Zumbuehl, A. Shear-stress sensitive lenticular vesicles for targeted drug delivery. Nat. Nanotechnol. 2012, 7, 536543.

(101) Wang, J.; Kaplan, J. A.; Colson, Y. L.; Grinstaff, M. W. Mechanoresponsive materials for drug delivery: Harnessing forces for controlled release. Adv. Drug Delivery Rev. 2017, 108, 68-82.

(102) Saxer, T.; Zumbuehl, A.; Müller, B. The use of shear stress for targeted drug delivery. Cardiovasc. Res. 2013, 99, 328-333.

(103) Allen, T. M.; Cullis, P. R. Liposomal drug delivery systems: from concept to clinical applications. Adv. Drug Delivery Rev. 2013, 65, $36-48$.

(104) Sackmann, E. The seventh Datta Lecture. Membrane bending energy concept of vesicle- and cell-shapes and shape-transitions. FEBS Lett. 1994, 346, 3-16.

(105) Bernard, A. L.; Guedeau-Boudeville, M. A.; Marchi-Artzner, V.; Gulik-Krzywicki, T.; di Meglio, J. M.; Jullien, L. Shear-induced permeation and fusion of lipid vesicles. J. Colloid Interface Sci. 2005, $287,298-306$.

(106) Cheng, C.; Helderman, F.; Tempel, D.; Segers, D.; Hierck, B.; Poelmann, R.; van Tol, A.; Duncker, D. J.; Robbers-Visser, D.; Ursem, N. T.; van Haperen, R.; Wentzel, J. J.; Gijsen, F.; van der Steen, A. F.; de Crom, R.; Krams, R. Large variations in absolute wall shear stress levels within one species and between species. Atherosclerosis 2007, 195, 225-235.

(107) Neuhaus, F.; Mueller, D.; Tanasescu, R.; Balog, S.; Ishikawa, T.; Brezesinski, G.; Zumbuehl, A. Synthesis and Biophysical Characterization of an Odd-Numbered 1,3-Diamidophospholipid. Langmuir 2018, 34, 3215-3220.

(108) Szebeni, J.; Moghimi, S. M. Liposome triggering of innate immune responses: a perspective on benefits and adverse reactions. J. Liposome Res. 2009, 19, 85-90.

(109) Wibroe, P. P.; Anselmo, A. C.; Nilsson, P. H.; Sarode, A.; Gupta, V.; Urbanics, R.; Szebeni, J.; Hunter, A. C.; Mitragotri, S.; Mollnes, T. E.; Moghimi, S. M. Bypassing adverse injection reactions to nanoparticles through shape modification and attachment to erythrocytes. Nat. Nanotechnol. 2017, 12, 589-594.

(110) Bugna, S.; Buscema, M.; Matviykiv, S.; Urbanics, R.; Weinberger, A.; Meszaros, T.; Szebeni, J.; Zumbuehl, A.; Saxer, T.; Muller, B. Surprising lack of liposome-induced complement activation by artificial 1,3-diamidophospholipids in vitro. Nanomedicine 2016, $12,845-849$.

(111) Buscema, M.; Matviykiv, S.; Mészáros, T.; Gerganova, G.; Weinberger, A.; Mettal, U.; Mueller, D.; Neuhaus, F.; Stalder, E.; Ishikawa, T.; Urbanics, R.; Saxer, T.; Pfohl, T.; Szebeni, J.; Zumbuehl, A.; Müller, B. Immunological response to nitroglycerin-loaded shearresponsive liposomes in vitro and in vivo. J. Controlled Release 2017, 264, 14-23.

(112) Korin, N.; Kanapathipillai, M.; Matthews, B. D.; Crescente, M.; Brill, A.; Mammoto, T.; Ghosh, K.; Jurek, S.; Bencherif, S. A.; Bhatta, D.; Coskun, A. U.; Feldman, C. L.; Wagner, D. D.; Ingber, D. E. Shear-Activated Nanotherapeutics for Drug Targeting to Obstructed Blood Vessels. Science 2012, 337, 738-742.

(113) Molloy, C. P.; Yao, Y.; Kammoun, H.; Bonnard, T.; Hoefer, T.; Alt, K.; Tovar-Lopez, F.; Rosengarten, G.; Ramsland, P. A.; van der Meer, A. D.; van den Berg, A.; Murphy, A. J.; Hagemeyer, C. E.; Peter, K.; Westein, E. Shear-sensitive nanocapsule drug release for site-specific inhibition of occlusive thrombus formation. J. Thromb. Haemostasis 2017, 15, 972-982. 